\title{
Minute Times Micromole Per Liter Per Kilogram
}

National Cancer Institute

\section{Source}

National Cancer Institute. Minute Times Micromole Per Liter Per Kilogram. NCI

Thesaurus. Code C112341.

Minutes times micromoles per liter, divided by kilograms. 